Archives

16 | 1996

Pour une histoire comparée du vœu

\title{
Le sens du vœu dans les ordres chevaleresques européens du moyen âge
}

Jesus D. Rodriguez Velasco

\section{(2) OpenEdition}

Journals

Édition électronique

URL : http://journals.openedition.org/ccrh/2649

DOI : $10.4000 /$ ccrh.2649

ISSN : $1760-7906$

Éditeur

Centre de recherches historiques - EHESS

Édition imprimée

Date de publication : 16 avril 1996

ISSN : 0990-9141

Référence électronique

Jesus D. Rodriguez Velasco, «Le sens du vœu dans les ordres chevaleresques européens du moyen âge », Les Cahiers du Centre de Recherches Historiques [En ligne], 16| 1996, mis en ligne le 27 février 2009, consulté le 20 avril 2019. URL : http://journals.openedition.org/ccrh/2649; DOI : 10.4000/ ccrh.2649

Ce document a été généré automatiquement le 20 avril 2019

Article L.111-1 du Code de la propriété intellectuelle. 


\title{
Le sens du vœu dans les ordres chevaleresques européens du moyen âge
}

\author{
Jesus D. Rodriguez Velasco
}

\section{NOTE DE L'ÉDITEUR}

Traduit par Amaia Arizaleta et Alain Boureau.

1 L'aventure constitue l'essence du chevalier. Entre le chevalier et l'aventure se noue une liaison intime, celle de l'homme et de sa vie. Mais les aventures ne sont pas toutes identiques. La littérature médiévale distingue - en liaison avec une évolution de la conception de l'aventure - l'aventure épique de l'aventure courtoise. L'aventure épique émane de la volonté et des vertus de celui qui l'entreprend. Roland accompagné des onze paladins, les pairs de France, choisit d'entrer en Espagne afin de mener à bien la grandiose aventure de la chrétienté ${ }^{1}$. Guillaume au courbe nez, irrité par l'ingratitude du roi Louis, part décidément à la conquête d'Orange, de Nîmes, de Narbonne et d'autres endroits ${ }^{2}$. Le Cid, mu par des considérations politiques, prend la décision de construire sa propre vie, son propre pouvoir, en conquérant toutes les forteresses sarrasines qu'il trouvera sur son chemin, d'Alcocer jusqu'à Valence ${ }^{3}$. Dans la carrière héroïque, le chevalier épique trouve sa satisfaction dans l'expression de son propre courage, qu'il maîtrise assez pour parachever ses desseins.

2 En revanche, dans le monde courtois, l'aventure est le fruit du hasard. Le chevalier ne cherche pas l'aventure. Elle s'offre à lui. Il ne l'accomplit que si elle lui a été réservée. Dans le cas contraire, il essuiera un échec d'autant plus cuisant que cette aventure était réservée à un autre chevalier. Keu, Gauvain et Lancelot partent avec une même intention : libérer les habitants du royaume de Logres faits prisonniers par Bademagus, roi de Gorre ; seul Lancelot, néanmoins, atteint son but, perdant son honneur, lors du voyage en charrette, alors que Keu aggrave la situation en perdant la reine et que Gauvain arrive en retard $^{4}$. De même, tous les chevaliers de la Table Ronde entreprennent la quête du saint 
Graal après un serment solennel ; seuls trois d'entre eux trouvent le Graal et seul Gallaaz, le meilleur chevalier du monde, obtient pleinement la grâce du saint Vase ${ }^{5}$. Les autres ne réunissent plus les conditions nécessaires à cette grâce.

C'est précisément dans l'aventure courtoise que l'on trouve le vœu utilisé comme procédé pour contrecarrer le hasard de l'aventure. On pourrait penser que le vœu constitue un recours épique, en tant qu'acte volontaire si fort qu'il peut vaincre les contraintes du hasard. Mais le vœu représente une médiation, non une finalité; il ne constitue pas une aventure, mais le moyen d'accomplir cette aventure. Ainsi Keu, sénéchal de la cour $d^{\prime}$ Arthur, exploite sa position politique et l'immense force du don contraignant ${ }^{6}$ pour réussir à s'intégrer à l'aventure. Les compagnons du Graal font serment d'entreprendre une quête dont ils connaissent bien l'échec probable: ils promettent de ne pas se présenter à la cour pendant un an et un jour, jusqu'à ce qu'ils aient trouvé le Graal. En participant aux conditions de l'aventure, Keu risque son statut privilégié, son appartenance au monde courtois. Au cas où ses calculs n'auraient pas donné le fruit souhaité, il aurait disparu du scénario courtois, dans le néant extérieur à la Table Ronde ${ }^{7}$, seul groupe civilisé du monde chevaleresque. Pour les compagnons du Graal, l'absence prolongée, loin de la cour arthurienne, entraîne un manque de protection politique et morale, car la cour représente le centre de l'ordre hors duquel n'existe que la crise chevaleresque ${ }^{8}$.

4 Voici donc une manifestation littéraire, un acte de médiation pour dominer le hasard, qui peut et doit conduire à la finalité souhaitée de l'aventure, c'est-à-dire, par excellence, au fait chevaleresque. Mais cette forme de volonté impose de sérieuses difficultés à celui qui veut s'y soumettre, car, dès ce moment, le chevalier est obligé de se mettre en dehors des conventions d'une société codifiée, de façon à pouvoir agir conformément à sa fonction.

5 Le vœu chevaleresque n'a aucun sens hors du monde courtois, où il est associé à l'ordo9. Les formes les plus anciennes du vœu chevaleresque sont donc associées à cet élément d'ordre et de civilisation qu'est le monde arthurien, au travers de la Table Ronde. Mais d'autres formes de contrat volontaire, comme le serment de chevalerie (qui nous intéressera plus loin), ne représentent pas que des motifs littéraires: dans le monde courtois, le serment constitue un acte essentiel qui fait partie des conditions d'entrée dans l'ordre chevaleresque, lors de l'adoubement ${ }^{10}$.

6 Or la Table Ronde représente elle-même un ordre chevaleresque qui, comme les autres ordres militaires ou religieux, ou comme l'ordre de chevalerie en général, n'est pas réglé par des lois imposées par le roi ou par d'autres législateurs; mais qui se soumet à des coutumes établies par un serment ou un vœu. L'origine de la chevalerie, considérée comme un sacrement par de nombreux auteurs, de Jean de Salisbury ${ }^{11}$ à Ramon Llull ${ }^{12}$ ou don Juan Manuel ${ }^{13}$, se fonde sur le serment ou le vœu d'initiation qui permet à l'écuyer de devenir chevalier de plein droit. Si nous retournons au monde arthurien, nous pouvons observer que la décision la plus immédiate du roi Arthur, après la réception de la Table Ronde, relève précisément du vœu : personne ne pourra s'asseoir à table si une aventure ne lui est pas arrivée, si elle n'est pas livrée à tous les chevaliers pendant les fêtes solennelles, comme le jour de Pentecôte :

«Et li rois comande que les napes soient mises, car il est tens de mengier, ce li est avis. "Sire, fet Kex, li seneschaux, se vos asseez ja au disner, il m'est avis que vos enfraindroiz la costume de ceanz. Car nos avons veu toz jorz que vos a haute feste n'asseiez a table devant que aucune aventure fut en vostre cort voiant toz les barons de vostre ostel. » «Certes, fet li rois, Kex, vos dites voir. Ceste costume ai je toz jorz tenue et la tendrai tant come je porrai. Mes je avoie si grans joie de 
Lancelot et de ses cousins qui estoient venu a sort sain et haiti, qu'il ne me sovenoit

de la costume. ${ }^{14}$ "

7 Le vœu prononcé par le roi et par chacun des compagnons, volontairement, mais en imitation du roi, s'établit comme une coutume, la « costume de ceanz », comme un droit coutumier, qui ne peut être enfreint sans menacer la confraternité. Le vœu se manifeste comme un système de solidarité corporative, au même niveau que la loi. Selon Dominique Boutet, «Ce n'est pas un roi qui édicte ces règles; elles ne sont pas non plus le fait d'une réflexion et d'une décision collectives, dont le roi ne serait qu'un garant. Leur source est atomisée, et le vœu de chacun s'impose à tous : Arthur, les chevaliers de la Table Ronde, Gauvain surtout, Guenièvre enfin, contribuent séparément à cette codification de l'activité chevaleresque et curiale ${ }^{15}$. "

8 Ce fait a été assez rapidement perçu comme une découverte heureuse en vue de la construction d'une chevalerie orientée vers la figure du roi, puis du prince. Elle valorisait chacun des chevaliers. Ce qui, en principe, se présente comme une action spontanée, comme le signale $\mathrm{D}$. Boutet à propos de la première fête célébrée autour de la Table Ronde, devient, par son utilité et sa solidité, un recours codifié qui s'impose par le biais du serment solennel, au travers de la force de la loi, de la « costume de ceanz ».

L'Ordre de l'Écharpe (Orden de la Banda) est la première congrégation chevaleresque laïque et royale instituée en Europe, la fondation de celle-ci revient à Alphonse XI de Castille, qui ne put la mettre en œuvre qu'en 1330, après avoir réglé quelques problèmes administratifs ${ }^{16}$; mais il y songeait déjà en 1325 . Peu après la fondation de l'ordre, le roi Alphonse fit dresser un règlement détaillé, où il exprimait avec une précision arithmétique chacun des droits et des devoirs du chevalier de l'Écharpe. Malgré ce juridisme, l'appartenance à l'ordre demeurait strictement volontaire. Pour se joindre à l'ordre, le chevalier devait faire voir sa valeur au travers de procédures chevaleresques. Il devait aussi prononcer un serment solennel semblable aux vœux chevaleresques que l'on trouve dans la littérature européenne.

10 Le serment de l'Ordre de l'Écharpe est divisé, essentiellement, en deux parties. L'une d'entre elles est liée aux systèmes de loyauté chevaleresque du monde courtois, dont le centre est le roi qui, dans ce cas-ci, est un roi-maître, ainsi que les frères de l'Écharpe :

«Vos avedes a jurar estas dos cosas: la primera, que en toda vuestra vida que seades en serviçio del rey e que seades siempre vasallo del rey o de alguno de sus fijos; pero si acaesçiese que vos partiésedes del rey o de alguno de sus fijos, quel enviedes la Vanda al rey et que nunca la podades pedir jamás para que vos la dén; [...] Otrosy, la segunda que avedes de jurar es que amedes a los cavalleros de la Vanda, asi como a vuestros hermanos, et que non desayudedes a otro cavallero de la Vanda salvo sy fuese ayudando a padre o a hermano. $»^{17}$

11 Ce serment appartient à la structure la plus élémentaire de la chevalerie. Il s'agit, sous toutes ses formes, d'un vœu de loyauté chevaleresque. La seule différence par rapport au serment de la chevalerie, c'est que le vœu de l'Écharpe peut être révoqué ; alors que le serment général de la chevalerie est une sorte de sacrement qui imprime un " caractère ", comme le baptême ou l'ordre sacerdotal, l'appartenance à l'Ordre de l'Écharpe est volontaire et peut être dénoncée par le chevalier. En fait, au lieu de faire partie d'un système juridique, comme le serment de chevalerie, il fait partie d'un code chevaleresque conventionnel dont on trouvera les caractéristiques réglées dans l'Ordonamiento de la Banda (Charte de l'Écharpe). 
12 Il existe, par ailleurs, une deuxième partie, plus intimement liée à la structure des vœux chevaleresques généralement transmis par la littérature. Afin d'intégrer l'Ordre de l'Écharpe par une volonté propre, il est nécessaire de se soumettre à un processus médiateur dont la difficulté constitue la valeur fondamentale. Premièrement, l'aspirant devra jouter contre deux chevaliers de l'Écharpe, l'un après l'autre, sans qu'il y ait place pour aucune sorte de répit; dans le cas où l'aspirant sortirait vainqueur de l'épreuve, il devrait faire à nouveau la preuve de sa valeur pendant le premier tournoi qui aura lieu en Castille, auquel participeraient des chevaliers de l'Écharpe :

« [...] que entre y que tome con dos, uno a uno, e que faga dos venidas con cada uno, e esto que sea en un medio día. Y si le acaesçiere quel cayere el espada de la mano sin quebrarle la manzana e sin quebrarle el espada, o si cayere del cavallo sin caer el cavallo con él, que sea vençido e que no pueda traer banda, así como aquel que se le caye el espada de la mano y caye del cavallo allí do lo ha menester. E si le acaesçiere quel cayere el espada de la mano o cayere del cavallo según dicho es a alguno de los que ante trayan la banda, que aya vençido al uno, e si amos, que sean vençidos amos e quel den la banda según dicho es de suso. E que faga aquel juramento que an fecho los cavalleros de la Vanda e que la puedan traer dende adelante ${ }^{18}$.

Les règles de l'ordre de l'Écharpe embrassent de nombreuses obligations de type courtois et chevaleresque. A l'image de la Table Ronde, les chevaliers doivent défendre en premier lieu leur roi et son royaume; en deuxième lieu, des liens de solidarité chevaleresque se créent entre les chevaliers; enfin, le chevalier de l'Écharpe devra consacrer sa voix et son corps à la défense de toute femme qui puisse avoir besoin d'aide. A l'image encore de la Table Ronde, les chevaliers de l'Écharpe doivent obligatoirement être présents à tous les tournois qui seront organisés à la cour et, en plus, ils devront se réunir autour du roi à l'occasion de certaines fêtes et, surtout, à la Pentecôte. Toutes ces obligations sont inhérentes au serment de chevalier de l'Écharpe puisque, en dernière instance, celui-ci devra devenir responsable, par un autre serment, de tout ce qui est contenu dans l' Ordenamiento de la Banda, livre conservé dans la chambre du roi et qui pouvait être consulté en cas de doute ${ }^{19}$.

L'idée d'un ordre chevaleresque signifie la hiérarchie de la chevalerie: elle induit une chevalerie dans la chevalerie. Le serment de cette nouvelle chevalerie ne signifie pas une renonciation; mais il désigne la concrétion de ses objectifs, ainsi que des conditions qui lui permettront d'atteindre sa finalité. Ce serment entraîne une double reconnaissance. En premier lieu, l'acceptation de la société politique chevaleresque, ce qui n'était pas évident dans l'Europe pré-monarchique ; deuxièmement, l'acceptation d'un code moral, le code chevaleresque, qui prend la forme d'un engagement de la défense de l'ordre social à travers les principes de la courtoisie.

15 Cette chevalerie réglée selon les modèles courtois, flagrante héritière de la chevalerie littéraire, s'est bientôt étendue au reste des cours européennes, où un grand nombre d'ordres de ce genre virent le jour, chacune avec ses statuts et ses vœux corporatifs. Rappelons pour mémoire les Ordres de la Jarretière, de l'Étoile, de la Toison d'Or, ordres monarchiques fondés à l'image de l'Ordre de l'Écharpe. Mais ce ne sont pas les seuls : les nobles de plus haut rang ont aussi créé les leurs. L'un des plus intéressants, parce qu'il avait des statuts très éloquents, est celui de La Dame Blanche à l'Écu Vert, fondée par Jean le Maingre, Boucicaut, en 1400. L'organisation de cet ordre chevaleresque fut l'œuvre du maréchal français et de douze autres compagnons qui, ensemble, rédigèrent un statut contenant un vœu d'obligation. La maîtrise de l'ordre de Boucicaut est, dans une large mesure, corporative: la coutume est établie par des articles que les treize associés 
contrôlent par leur vote. Le fait le plus digne d'attention est que, comme dans les coutumes fondatrices de la Table Ronde, c'est l'imitation du primus inter pares qui entraîne la constitution de l'ordre ${ }^{20}$.

16 La manifestation la plus fréquente du vœu chevaleresque a souvent lieu au sein de la corporation chevaleresque elle-même. Qu'il s'agisse de la Table Ronde, de l'Écharpe, de la Toison d'Or ou de toute autre corporation secondaire, mais détentrice d'une certaine solidité politique. Dans l'immense encyclopédie chevaleresque qu'est le Roman de Perceforest, on analyse soigneusement la généalogie entière du monde chevaleresque, depuis Alexandre le Grand. Après la victoire d'Alexandre sur Porus, le monde alexandrin se stabilise ; la première manifestation d'une telle stabilisation est un dîner des chevaliers qui, unis dans la fraternité chevaleresque, ont combattu les armées des barbares. Une table, autour de laquelle se réunit l'élite suprême de l'antique chevalerie, sert à établir la première série de vœux sur le faisan, oiseau très noble, qu'ils vont se partager pendant le repas $^{21}$.

L'idée avait été mise en relief peu d'années auparavant par Jacques de Longuyon qui, suivant le style du Roman d'Alexandre, avait composé une narration complémentaire à la troisième branche du Roman, avec laquelle ce texte a souvent été transmis. Il s'agit des célèbres Vœux du Paon ${ }^{22}$, qui ont donné lieu à une série de continuations et de corrections, comme le Restor du Paon de Brisebarre (Jean le Court) ${ }^{23}$ ou le Parfait du Paon, continuation du Restor, composé par Jean le Mote ${ }^{24}$. Le contenu de ces vœux est fondamentalement courtois, romanesque, centré sur des questions telles que celle de la hardiesse, du courage ou du secours aux femmes. C'est un « jurement » fanfaron des confrères de l'ordre qui ne se différencie pas des conditions établies par les ordres laïques monarchiques et nobiliaires dont nous avons parlés ${ }^{25}$. Mais, en revanche, le cycle du Paon et, spécialement, la partie due à Jacques de Longuyon, amènent un type de vœu chevaleresque qui éclaire la vraie fonction de ce vœu.

Je pense aux vœux chevaleresques dont les conditions ne sont pas courtoises, mais clairement princières, monarchiques et directement liées à l'action politique. Les attitudes comme celles qui sont enregistrées dans les Vœux $d u H_{\text {éron }}{ }^{26}$ ou les Vœux $d u$ Faisan $^{27}$ dans les cours anglaise et bourguignonne nous fournissent un échantillon de l'évolution des vœux corporatifs dont on a hérité, non seulement une vision littéraire de la fonction guerrière, mais aussi une attitude politique de type monarchique, car cette évolution apparaît dans des cours princières qui cherchent désespérément leur image étatique ${ }^{28}$. Dans ces deux vœux, on exploite la capacité, annoncée par la figure d'Arthur, de convertir un vœu en coutume, ainsi que son corrélat : l'acceptation de ces conditions par les chevaliers proches entraîne la reconnaissance des règles de ce monde, de cette congrégation. Les particularités de chacun de ces vœux nous renvoient, cependant, à des réalités différentes. Les Vœux du Héron, probablement postérieurs à la création de l'Ordre de la Jarretière, sont l'écho de la traitrise de Robert d'Artois et, bien qu'ils témoignent de la solidarité des chevaliers anglais autour de leur roi, il n'en est pas moins certain qu'ils formulent une critique de l'entreprise guerrière qui avait mené à la guerre de Cent Ans ${ }^{29}$. Les Vœux du Faisan, d'un autre côté, montrent la tension qui existait entre la Bourgogne et la France dans la deuxième moitié $\mathrm{du} \mathrm{xv}^{\mathrm{e}}$ siècle : le duc Philippe de Bourgogne s'arroge le droit d'établir un vœu de croisé, et de l'imposer à chacun de ses chevaliers mais, en même temps, il fait dépendre son vœu du départ en croisade du roi de France; d'un côté, il revendique sa primauté dans le duché; de l'autre, il met en évidence sa dépendance relative du royaume de France. 
Concluons : le vœu chevaleresque est un vœu d'imitation, une condition répétitive qui représente, en même temps, un modèle de conduite, une consuetudo, et une acceptation du pouvoir du titulaire de l'ordre chevaleresque. Je soulignerai que le vœu n'a de sens que dans un monde où l'ampleur de la chevalerie est restreinte, contrôlée par une figure princière autour d'une corporation réglementée : la Table Ronde est réglée par une figure princière, qui impose le hasard d'une aventure visible aux yeux de tous; l'Écharpe, de plus étroite ordonnance, fixe le type d'aventure que le chevalier jurera; la table autour de laquelle se réunissent les compagnons d'Alexandre le Grand sert de support aux Vœux du Paon, qui réaffirme que l'aventure vient de se terminer ; la Jarretière, enfin, dont l'origine est à chercher dans le serment des chevaliers anglais après l'intervention de Robert d'Artois (Vœux du Héron), aux côtés de l'Ordre de la Toison d'Or, où l'on prononce les Vœux $d u$ Faisan, promet de commencer une aventure, dont l'acte de médiation et de contrôle est conditionné par le vœu.

Le vœu chevaleresque est donc, au sein de l'ordre, un acte collectif qui encadre un contrat de solidarité avec les conditions établies par celui qui a fondé l'ordre, qui impose la répétition de ce contrat à ses membres. Naturellement, l'un des effets immédiats de ce vœu est l'acceptation du commandement de celui qui a imposé son émission; le vœu individualise le promoteur au-dessus de ceux qui sont statutairement obligés de le répéter. Tel est l'effet final du vœu chevaleresque dans l'ordre, et la grande adresse du monde arthurien, c'est la création d'un monde chevaleresque dirigé par un monarque, un prince dont les actes deviennent coutume. Dans la mesure où il n'est pas possible d'enfreindre la coutume, celle-ci finit par être loi.

\section{NOTES}

1. Chanson de Roland

2. La Chanson de Guillaume, La Prise d'Orange, Le Charroi de Nîmes.

3. Cantar de Mio Cid.

4. Chrétien de Troyes, Le Chevalier à la charrette.

5. La Quête du saint Graal, version en prose de la vulgate arthurienne.

6. Le don contraignant est, de fait, l'un des moteurs de l'aventure. Seul celui qui demande le don sait ce qu'il implique, alors que celui qui l'accorde le fait à l'aveuglette, sans savoir ce qu'il est en train d'accorder. D'où la crise. Aussi, les consignes du hasard en sont augmentées. Erich Köhler a analysé ceci, dans la perspective de la sociologie littéraire, dans La aventura caballeresca. Ideal y narrativa en la cortés, Barcelona, Sirmio, 1990 (l'édition allemande originale est de 1957, révisée en 1970) ; Jean Frappier, "Il motivo del «don contraignant»", maintenant dans Maria Luisa Meneghetti, éd., Il romanzo, Bolonia : Il Mulino, Strumenti di Filologia Romanza, 1992, p. 347-387.

7. La matière de Bretagne, en général, tend à montrer que les Bretons sont le peuple civilisé par antonomase. L'histoire d'Arthur est exemplaire dans ce cas-ci, puisqu'il affronte non seulement les peuples les plus sauvages, comme les saxons du roi Ryon (Histoire de Merlin du pseudo-Boron), mais aussi quand il fait preuve d'évidente hostilité envers des peuples qui, jadis, avaient pratiqué la civilisation et qui, même, sont [prétendument. Ajout A.A.] à l'origine de la civilisation bretonne, comme c'est le cas des Romains : l'indéniable maladresse de l'empereur romain Ponce 
Antoine dans le Lancelot de la vulgate arthurienne est un échantillon palpable de ce fait, dont la finalité est de montrer la réalité d'une primitive translatio imperii studiique vers les côtes bretonnes. Finalement, le cycle arthurien affine encore la voie, lorsqu'il organise la civilisation autour de la figure monarchique-chevaleresque d'Arthur, placée sous l'ordre parfait, aussi symbolique que politique, de la Table Ronde qui, à tous les effets, est un ordre chevaleresque dont le grand maître est Arthur lui-même.

8. L'analyse détaillée d'Erich Köhler (L'aventure chevaleresque..) montre justement que, dans la structure du roman courtois, le départ, loin de la cour d'Arthur, entraîne l'élément de crise dans le roman, crise qui finit quand le héros retourne à la cour. Köhler montre que la succession crise/ ordre n'est ni linéaire ni unique, mais qu'elle est le fruit d'une tension, qui tient, d'un côté, à la crise chevaleresque provoquée par la sortie vers l'aventure et, après un moment d'ordre, à l'apparition d'une deuxième crise provoquée par la sécurité politique et morale, la recreantisse, qui est l'état antichevaleresque par définition, puisqu'il n'y a pas la place pour l'aventure en elle.

9. Dès ses origines, le vœu, n'importe quel genre de vœu, a son sens à l'intérieur d'une discipline vitale. Il serait simple d'argumenter cette affirmation à partir de la base du plus ancien vœu du monde chrétien, qui est le vœu clérical : au concile de Nicée, en 325, on établit que tous ceux qui embrasseraient l'état clérical prononceraient des vœux solennels, d'autant plus privatifs de la vie sensuelle que les ordres reçus seraient élevés.

10. Mais ceci représente un problème, cependant, car l'ordre chevaleresque restreint n'existe pas, comme le montrent les études de Jean Flori, L'idéologie du glaive. Préhistoire de la chevalerie, Genève, Droz, 1983, et L'Essor de la chevalerie, Genève, Droz, 1986.

11. Policraticus, livre VI.

12. Llibre de l'orde de cavalleria, cap. IV, Barcelone, éd. Marina Gustà, 1980.

13. Libro del cavallero e del escudero, cap. XVIII, Madrid, éd. José Manuel Blecua, 1982, vol. 1. Pour toutes ces questions, je me permets de renvoyer à mon livre Don Juan Manuel ante a caballeria cortés, Londres, sous presse.

14. La Queste del Saint Graal, Paris, éd. A. Pauphilet, $1984^{2}$, p. 4-5.

15. D.Boutet, Charlemagne et Arthur, ou le Roi imaginaire, Genève, 1992, p. 339. Sur ce point, D.Boutet souligne aussi la différence existante entre le droit féodal propre à l'épique et des coutumes votives, initiales, spécialement désignées et qui participent à la création du système de règles du monde courtois arthurien.

16. Peter Linehan, "Alfonso XI of Castile and the Arm of Santiago (with a note on the Pope's foot », dans P. Weimar et A. Garcia y Garcia, Miscellanea Domenico Maffei. Historia. Jus. Studium J., Francfort, 1993.

17. «Vous devez jurer ces deux choses : la première, que vous serez toute votre vie au service du roi et que vous serez toujours vassal du roi ou de l'un de ses enfants; mais si vous veniez à vous éloigner du roi ou de l'un de ses enfants, que vous enverrez l'écharpe au roi et que vous ne pourrez jamais plus la redemander, [...] Par ailleurs, la deuxième chose que vous devez jurer est que vous aimerez les chevaliers de l'Écharpe, comme vous aimez vos propres frères, et que vous ne refuserez pas l'aide à un autre chevalier de l'Écharpe, sauf si c'est pour aider votre père ou votre frère ». (Trad. d'A.A.) Ordenamiento de la Banda, chapitre II, toujours cité selon l'édition (sujette à discussion) d'Alfonso de Ceballos-Escalera y Gila, La Orden y Divisa de la Banda Real de Castilla, Madrid: Prensa y Ediciones Iberoamericanas, Colección «Persevante Borgoña », 1993, p. 58-59.

18. "[...] qu'il entre et qu'il joute contre deux chevaliers, l'un après l'autre, et qu'il fasse deux combats avec chacun, et que ceci dure une demi-journée. Et s'il venait à lâcher l'épée sans briser le pommeau ni l'épée, ou sil venait à tomber du cheval sans que le cheval tombe avec lui, qu'on le considère comme vaincu et qu'il ne puisse pas porter l'écharpe, comme celui qui lâche l'épée et qui tombe du cheval là où il en a besoin. Et si l'un de ceux qui portaient l'écharpe venait à lâcher l'épée ou à tomber, comme on l'a dit, qu'on considère qu'il [l'aspirant. N.d.t.] a vaincu celui-là et 
s'il s'agit de deux chevaliers, qu'on considère que tous les deux sont vaincus, et qu'on donne l'écharpe, comme on l'a dit plus haut. Et qu'il prononce le serment que les chevaliers de l'Écharpe ont prononcé, et qu'il puisse la porter dorénavant. " (Trad. A. A.) Op. cit, chap. III, p. 59-60. Je signale que ces conditions ne sont nécessaires que lorsqu'on désire faire partie de l'ordre de l'Écharpe : «Ces conditions n'ont de valeur que chez ceux qui voudront faire partie de l'Écharpe, mais si d'autres veulent jouter ou tournoyer avec les chevaliers de l'Écharpe, pour mettre à l'épreuve leur chevalerie, et non pas pour gagner l'écharpe, qu'il puissent le faire sans ces conditions que l'on vient d'énumérer, si les autres chevaliers sont d'accord » (trad. A. A.).

19. Le plus ancien manuscrit de l'Ordenamiento de la Banda se trouve à la Bibliothèque nationale de Paris, esp. 33. Il fut probablement écrit à la fin de la vie d'Alphonse XI. Il s'agit d'un manuscrit in folio, en parchemin et historié avec des capitales en or dans le prologue et à l'encre rouge et bleu pour le reste du texte. Le chapitre où l'on a noté les noms des chevaliers de l'Écharpe est suivi de plusieurs feuillets blancs, ce qui fait songer à un acte permanent de l'ordre, où l'on aurait prévu l'association de nouveaux membres dont on écrirait les noms par la suite. Les manuscrits postérieurs de l'Ordenamiento, tout comme le II Ordenamiento de la Banda, émis probablement par Jean $I^{\text {er }}$ de Castille, présentent des listes différentes de membres.

20. Le livre des fais du bon messire Jehan le Maingre, dit Boucicaut, Mareschal de France et Gouverneur de Jennes, ed. de Denis Lalande, Genève, Droz, "Textes littéraires français ", 331, 1985, lib. I, chap. XXXVIII-XXXIX.

21. Roman de Perceforest, livre I, ed. J..H..M. Taylor, Genève, Droz, « Textes littéraires français ", 279, 1979.

22. R.L.G. Ritchie éd., dans The Buick of Alexander, Edinbourgh-Londres, Scottish Texts Society, 1921-1929.

23. R. Carey éd., Genève, Droz, « Textes littéraires français », 119, 1966.

24. R. Carey, Chapel Hill, University of North Carolina Press, 1972.

25. La tradition du gab, ou vœu fanfaron, est bien assise dans la littérature épique burlesque, laquelle est probablement contemporaine des romans arthuriens qu'on appelle "postclassiques ». Elle constitue, peut-être, la réponse aux spectaculaires vœux et conditions des textes arthuriens (Victoria Cirlot, «La estética postclásica en los romans artúricos en verso del siglo XIII ", dans "Studia in honorem prof. Martin de Riquer » Barcelone, Quaderns Crema, 1991, vol. IV, p.381-400. Citons, comme exemple, le plus ancien de ces textes, le Pèlerinage de Charlemagne, où l'on trouve les gabs des douze pairs de France.

26. Édition paléographique de G. Bertoni, selon le ms. 323 de Berne, Archivum Romanicum, 5, 1921, p. 426-436.

27. Il existe deux récits des Vœux du Faisan, celui d'Olivier de la Marche et celui de Matthieu d'Escouchy ; leurs plus pertinents fragments ont été récemment éditées par Colette Beaune dans le volume préparée par Danielle Régnier-Bohler, Splendeurs de la cour de Bourgogne, Paris : Robert Laffont, Bouquins, 1995, p. 1129-1192.

28. L'évolution de la pratique politique vers la constitution de l'état a été exposée par Bernard Guenée, Occident aux XIV et XV siècles. Les états, Paris : PUF, « Nouvelle Clio », 19914 ; la gestation des idées politiques qui ont permis cette évolution fait l'objet d'une étude de Jacques Krynen,

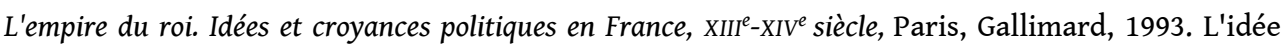
d'un régime étatique fondée sur l'idéologie chevaleresque est commun à Florence, Bourgogne et Castille au Xve siècle, comme j'ai tenté de l'exposer dans mon livre El debate sobre la caballería en el siglo $x v$, Valladolid, Junta de Castilla y León, 1996. La recherche du système de références de cet état chevaleresque a ses racines dans l'idée selon laquelle la chevalerie médiévale est l'héritière directe de la chevalerie romaine (je prépare actuellement une série de travaux concernant ce cadre référentiel, sous le titre générique de «Entre miles et eques. la culture courtoise européenne face à la chevalerie romaine »).

29. B.J. Whitting, « The Vows of the Heron ", Speculum, 20, 3, 1945, p. 261-278. 


\section{AUTEUR}

JESUS D. RODRIGUEZ VELASCO

Université de Salamanque 